\title{
ARTICLES
}

\section{Direct measurement of the branching ratio for the decay of the $\eta$ meson into two photons}

\author{
R. Abegg, ${ }^{1}$ R. Abela,${ }^{2}$ A. Boudard ${ }^{3}$ W. Briscoe,${ }^{4}$ M. Clajus,${ }^{5}$ J.-M. Durand,${ }^{6}$ A. Efendiev,${ }^{6,7}$ B. Fabbro, ${ }^{3}$ \\ P. Fuchs, ${ }^{6,1}$ M. Garçon, ${ }^{3, *}$ L. Lytkin, ${ }^{7}$ B. Mayer, ${ }^{3}{ }^{7}$ T. Morrison, ${ }^{4}$ B.M.K. Nefkens, ${ }^{5}$ V. Nikulin, ${ }^{3,6,8}$ J.-F. Pillot, ${ }^{3}$ \\ E. Tomasi-Gustafsson,,$^{6,3}$ D. White, ${ }^{5}$ and W.T.H. van Oers ${ }^{1}$ \\ ${ }^{1}$ TRIUMF, 4004 Wesbrook Mall, Vancouver, British Columbia, Canada V6T $2 A 3$ \\ ${ }^{2}$ Paul-Scherrer-Institut, CH-5232 Villigen, Switzerland \\ ${ }^{3}$ DAPNIA/SPhN, CEA-Saclay, 91191 Gif-sur-Yvette, France \\ ${ }^{4}$ Department of Physics, The George Washington University, Washington, DC 20052 \\ ${ }^{5}$ Department of Physics, UCLA, Los Angeles, California 90095-1547 \\ ${ }^{6}$ Laboratoire National Saturne, 91191 Gif-sur-Yvette, France \\ ${ }^{7}$ JINR, Laboratory of Nuclear Problems, 141980 Dubna, Moscow district, Russia \\ ${ }^{8}$ SPNPI, 188350 Gatchina, St. Petersburg district, Russia
}

(Received 12 May 1995)

\begin{abstract}
The first direct, precision measurement of the branching ratio for the decay of the $\eta$ meson into two photons, $B(\dot{\eta} \rightarrow \gamma \gamma) \equiv \Gamma(\eta \rightarrow \gamma \gamma) / \Gamma_{\text {tot }}(\eta)$, is presented. The systematic uncertainties are quite different than in previous indirect measurements. Using the SATURNE II proton beam and a liquid-deuterium target; the $\eta$ mesons were produced in the reaction $p d \rightarrow{ }^{3} \mathrm{He} \eta$ at $1.5 \mathrm{MeV}$ above threshold, and tagged by the detection of the recoil ${ }^{3} \mathrm{He}$ in the magnetic spectrometer SPES2. The two decay photons were detected in two BGO electromagnetic calorimeters located at $65^{\circ}$ left and right of the beam axis. A lead collimator placed in front of the right calorimeter defined the detector acceptance. The analysis yielded $B(\eta \rightarrow \gamma \gamma)=0.3949 \pm 0.0017$ (stat) \pm 0.0030 (syst).
\end{abstract}

PACS number(s): $13.40 . \mathrm{Hq}, 14.40 . \mathrm{Aq}$

\section{INTRODUCTION}

The branching ratio for the decay of the $\eta$ meson into two photons, $B(\eta \rightarrow \gamma \gamma) \equiv \Gamma(\eta \rightarrow \gamma \gamma) / \Gamma_{\text {tot }}(\eta)$, is pivotal in several respects: (i) together with the partial width $\Gamma(\eta \rightarrow \gamma \gamma)$ it determines the $\eta$ meson total decay width $\Gamma_{\text {tot }}(\eta)$, a quantity that cannot be measured directly with presently available experimental techniques; (ii) it is required for calculating the branching ratios of the electromagnetic $\eta$ decays for which $\eta \rightarrow \gamma \gamma$ is the intermediate state (the $\eta$ decay into one or two lepton pairs, or into a lepton pair and a photon); (iii) it determines the absolute magnitude of many $\eta$ production cross section measurements for which the $\eta$ meson is identified via its two photon decay.

The decay of a light pseudoscalar meson into two photons is described by the axial vector anomaly [1]. In the quark model, the calculation of the triangular loop involves a summation over the quark colors. The agreement between the calculation of $\Gamma\left(\pi^{0} \rightarrow \gamma \gamma\right)$ and the experimental value is often cited as the premier argu-

*Electronic address: garcon@phnx7.saclay.cea.fr ment in favor of the existence of three quark colors. The extension of the calculation to $\eta \rightarrow \gamma \gamma$ is straightforward, requiring only that the mixing of the $\mathrm{SU}(3)$ pseudoscalar octet, $\left(\eta_{8}\right)$ and singlet $\left(\eta_{1}\right)$ states is properly incorporated; this is accomplished using the mixing angle $\theta_{P}$. Denoting by $f_{\pi}, f_{8}$, and $f_{1}$ the pion, octet, and singlet decay constants, one finds

$$
\Gamma(\eta \rightarrow \gamma \gamma)=\frac{\alpha^{2} m_{\eta}^{3}}{96 \pi^{3}}\left[\frac{\cos \theta_{P}}{f_{8}}-\sqrt{8} \frac{\sin \theta_{P}}{f_{1}}\right]^{2} \simeq 500 \mathrm{eV}
$$

with $f_{1} \simeq f_{\pi} \simeq 132 \mathrm{MeV}, f_{8} \simeq 1.25 f_{\pi}$, and $\theta_{P} \simeq$ $-20^{\circ}$ [2]. Note that the contribution of the singlet state to $\Gamma(\eta \rightarrow \gamma \gamma)$ is significant, although the $\eta$ is mostly octet $\left(\eta_{8}\right)$, and that Shore and Veneziano [3] expressed reservation about the generalization of the axial anomaly calculation to $\eta_{1} \rightarrow \gamma \gamma$. The QCD (radiative) corrections to the triangular loop calculation are generally assumed to be negligible for light quark systems [4]. The experimental determination of $\Gamma(\eta \rightarrow \gamma \gamma)$ shows a discrepancy between two types of experiments: $\Gamma(\eta \rightarrow \gamma \gamma)=0.51 \pm 0.03$ $\mathrm{keV}$ for two-photon $\eta$ production from $e^{+} e^{-}$collisions, and $\Gamma(\eta \rightarrow \gamma \gamma)=0.32 \pm 0.05 \mathrm{keV}$ for $\eta$ production from a real photon in the Coulomb field of a nucleus, the socalled Primakoff production [5]. 
For a proper understanding of $B(\eta \rightarrow \gamma \gamma)$, the other main decays of the $\eta\left(\eta \rightarrow 3 \pi\right.$ and $\left.\eta \rightarrow \pi^{+} \pi^{-} \gamma\right)$ must be considered as well: the corresponding partial widths can be calculated using, respectively, chiral perturbation theory and effective chiral Lagrangians which incorporate vector mesons. The accuracy of these calculations, as well as the experimental and theoretical errors in $f_{8}, f_{1}$, and $\theta_{P}$ in Eq. (1), do not allow for a precise theoretical prediction for $B(\eta \rightarrow \gamma \gamma)$.

The hitherto accepted experimental value for the $\eta \rightarrow$ $\gamma \gamma$ branching ratio is [6]

$$
B(\eta \rightarrow \gamma \gamma)=0.388 \pm 0.005
$$

This value is primarily based on two experiments. The ratio $\Gamma(\eta \rightarrow$ neutrals $) / \Gamma_{\text {tot }}(\eta)=0.705 \pm 0.008$ was measured at CERN in an experiment [7] where the $\eta$ were produced in the reaction $\pi^{-} p \rightarrow \eta n$ and tagged by the detection of the neutron. The measurement of the number of $\eta$ produced relied on a subtraction of a large number of background events: the signal-to-background ratio was about $1 / 3$. Moreover, the background shape was characterized by a steep and varying slope. The shape of the background was estimated entirely using a Monte Carlo simulation and no discussion of the systematic uncertainties of this background subtraction was presented. The ratio $\Gamma(\eta \rightarrow \gamma \gamma) / \Gamma(\eta \rightarrow$ neutrals $)=0.549 \pm 0.004$ was measured at IHEP [8] ("neutrals" are in this case defined as the sum of the $2 \gamma, 3 \pi^{0}$, and $\pi^{0} \gamma \gamma$ channels). The experiment determined the ratios of the partial widths of the decays $\eta \rightarrow k \gamma$, for multiplicity $k$ up to 10 . The photons from the $\eta$ decays were detected in a lead glass calorimeter. The largest systematic uncertainties resulted from the difference in the $\eta \rightarrow \pi^{0} \pi^{0} \pi^{0}$ and $\eta \rightarrow \gamma \gamma$ detector acceptance and analysis efficiency. The determination of $B(\eta \rightarrow \gamma \gamma)$ has thus required to combine the results of these two experiments. In contradistinction, the experiment reported here is the first direct measurement of $B(\eta \rightarrow \gamma \gamma)$.

\section{EXPERIMENTAL ARRANGEMENT}

The experiment was conducted at the SPES2 tagged $\eta$ facility at the Laboratoire National Saturne, Saclay, France. The $\eta$ mesons were produced in the reaction $p d \rightarrow{ }^{3} \mathrm{He} \eta$ just above threshold. The measurement of $B(\eta \rightarrow \gamma \gamma)$ requires an accurate determination of the number of $\eta$ mesons produced and of the number of subsequent $\eta \rightarrow \gamma \gamma$ decays detected with known efficiency in a known solid angle. The first was accomplished using the SPES2 magnetic spectrometer which identifies the ${ }^{3} \mathrm{He}$ and measures its vector momentum. The second was done using a two-arm photon calorimeter to measure the energies and impact coordinates of the photons.

\section{A. Beam and target}

The proton beam from the Saturne II synchrotron was tuned to $1.5 \mathrm{MeV}$ above the $p d \rightarrow{ }^{3} \mathrm{He} \eta$ threshold en- ergy of $891.4 \mathrm{MeV}$. The synchrotron dipole field was stabilized to better than a gauss (out of $8310 \mathrm{G}$ ), resulting in a relative precision better than $\pm 80 \mathrm{keV}$ during the beam spill. In the few runs where this stability was not achieved, the analysis of the ${ }^{3} \mathrm{He}$ momentum spectrum as a function of time during the spill allowed monitoring the changes in the beam energy. The beam position on target was monitored between data runs through the use of insertable wire chambers in the beam line. During the data taking, the stability of the beam conditions was monitored by two telescopes, each consisting of three plastic scintillation counters located on either side of the beam, which viewed a 20- $\mu$ m-thick Mylar foil placed in the path of the incident proton beam, $970 \mathrm{~mm}$ upstream of the liquid-deuterium target. The beam intensity was limited typically to $3 \times 10^{9}$ protons per spill in order to have acceptable single rates in the photon detector. The beam spill was usually of $600 \mathrm{~ms}$ duration, repeated every $1.1 \mathrm{~s}$.

The liquid-deuterium target cell had two 15- $\mu \mathrm{m}$ titanium walls, fixed to an arc-shaped copper frame. The liquid thickness was about $6 \mathrm{~mm}$. The arc shape was chosen in order to reduce the amount of target and target wall materials between the $\eta \rightarrow \gamma \gamma$ vertex and the photon detector. The target cell was enclosed in $25 \mu \mathrm{m}$ of aluminized mylar for thermal insulation. The photons were going through 250- $\mu \mathrm{m}$ thick mylar lateral vacuum windows as well.

\section{B. The SPES2 spectrometer}

The recoil ${ }^{3} \mathrm{He}$ particles were detected around $0^{\circ}$ by the magnetic spectrometer SPES2 $[9,10]$. This spectrometer consists of a quadrupole magnet and two horizontal dipole magnets. The primary proton beam escapes between the two dipoles. The detection system consisted of three double-plane multiwire proportional chambers followed by two plastic scintillator planes " $A$ " and " $B$." The $A$ plane was segmented into six plastic scintillator paddles while the $B$ plane was formed by a plastic scintillator with photomultiplier (PM) tubes mounted at opposite ends. The ${ }^{3} \mathrm{He}$ particles were identified by the energy deposited in the $A$ plane and the time-of-flight between the $A$ and $B$ planes. All of the ${ }^{3} \mathrm{He}$ particles from $p d \rightarrow{ }^{3} \mathrm{He} \eta$ were accepted by SPES2. They were emitted within a small cone with half angle $1.2^{\circ}$ with respect to the beam axis and within a small $( \pm 3 \%)$ momentum band. The spectrometer momentum acceptance was much larger ( $\pm 10 \%$ defined by the $A$ plane).

The corresponding $\eta$ 's were emitted in a cone of half angle $6^{\circ}$ with a kinetic energy of $60 \mathrm{MeV}( \pm 15 \mathrm{MeV}$ at the base of the distribution). With the above specifications of beam intensity and target thickness, the number of tagged $\eta$ 's was about 30 per beam spill.

\section{The photon detector}

For $\eta \rightarrow \gamma \gamma$ decays which are symmetric with respect to the beam direction, each photon is emitted with an 
energy of about $300 \mathrm{MeV}$ at an angle of $65^{\circ}\left(90^{\circ}\right.$ in the rest frame of the $\eta$ ). The two arms of the photon detector were positioned at this angle, left and right of the beam axis, as illustrated in Fig. 1(a). The right photon detector arm consisted of a collimator with a circular aperture defining the acceptance for $\eta \rightarrow \gamma \gamma$ events, a plastic scintillator (" $V$ " counter) to identify charged particles and the calorimeter counters " $R$ " which measured the energy and impact position of a photon. The left photon detector arm consisted solely of the calorimeter counters " $L$."

Each calorimeter was composed of 61 bismuth germanate (BGO) scintillation counters of hexagonal cross section (with sides of $31.5 \mathrm{~mm}$ ) with a length of $200 \mathrm{~mm}$ (17.8 radiation lengths), stacked in an hexagonal arrangement [Fig. 1(b)]. The BGO counters, with their associated 5-cm PM and power supply circuitry, were developed at the University of Berne and used previously in an experiment at the Paul-Scherrer-Institut [11]. The shortterm PM gain variations as a function of time were monitored using test signals from an array of light-emitting diodes (LED's) coupled by optical fibers to each counter. The gain variations could reach $10 \%$ for the counters with the largest instantaneous rates $(8 \mathrm{kHz})$. Since the BGO light output has a decay time of about $300 \mathrm{~ns}$, pileup was a potential problem. It was demonstrated [12] that clipping can be used down to $100 \mathrm{~ns}$ of signal occupation time without significant loss of resolution. For this experiment, a pole-zero cancellation circuit, well suited to the exponential shape of the signal tail, was implemented to reduce the signal decay time by a factor 5 .

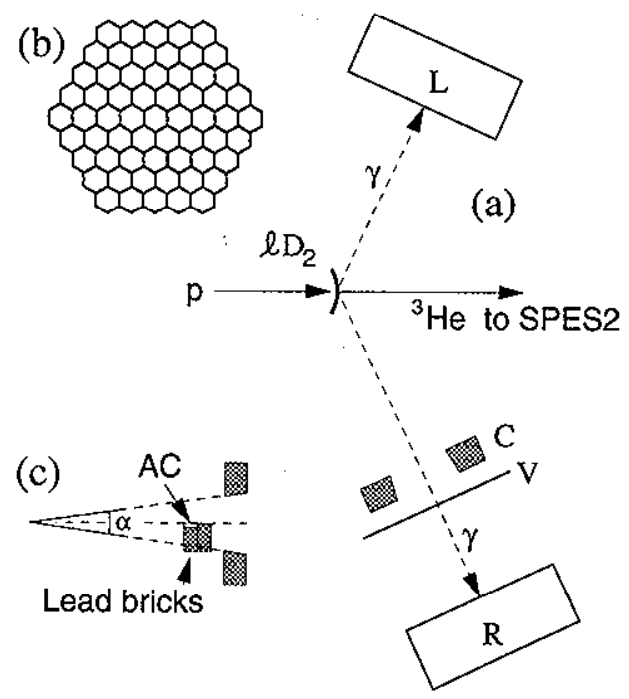

FIG. 1. The photon detector: (a) Top view (the arc-shaped target is not to scale): the calorimeters " $L$ " and " $R$ " measured the energies and angles of the outgoing photons; the collimator " $C$ " defined the acceptance of $R$; the " $V$ " counter detected charged particle background. (b) Front view of the $L$ and $R$ calorimeters, showing the stacking arrangement of the 61 BGO counters. (c) Cross sectional view of the collimator, with the definition of its aperture angle $\alpha$; the " $A C$ " counter and its supporting lead bricks defined an active collimator used only during some special runs.
Each calorimeter rested on a moveable platform. The distance from the $L$ calorimeter to the target could be varied between 540 and $730 \mathrm{~mm}$, and the $R$ calorimeter distance between 770 and $980 \mathrm{~mm}$. The nominal distance between the target and the $R$ calorimeter, $883 \mathrm{~mm}$, was a compromise between good angular resolution and minimization of loss of electromagnetic shower energy out of the sides of the calorimeter. The nominal distance between the target and the $L$ calorimeter, $653 \mathrm{~mm}$, was chosen such that this calorimeter accepted every photon from $\eta \rightarrow \gamma \gamma$ in association with a photon accepted by the $R$ calorimeter. The fraction of the energy of the electromagnetic shower which was lost out the sides of either calorimeter was less than $3 \%$.

Each calorimeter was placed inside a light-tight iron box that also ensured thermal control and shielded the PM's from the stray magnetic field of the spectrometer. Since the light output of BGO changes by approximately $1.5 \%$ per Kelvin, the temperature variations within the boxes were kept lower than $\pm 0.2 \mathrm{~K}$ by the use of regulated heating elements and fans.

Large plastic scintillation counters were placed above and under each calorimeter for defining a trigger for cosmic ray events going through the BGO counters. A simulation indicated that, with this geometry, the average energy deposit of cosmic rays was the same for every counter. Cosmic ray events acquired in between beam spills were used to calibrate the BGO counter amplitudes relative to each other.

Measurements were performed using in turn three collimators of different aperture. Each collimator was made of lead, $80-\mathrm{mm}$ thick (14.3 radiation lengths). Each had a precision-machined, tapered aperture of circular cross section. The acceptance of the collimators was a cone originating at the target and subtending an angle of $12^{\circ}$ (collimator 1 ), $15^{\circ}$ (collimator 2 ), or $18^{\circ}$ (collimator 3 ). Collimator 3 was used for most of the data taking.

Simulations indicated that $99 \%$ of the events which hit the lead collimator edge and resulted in an event in the $R$ calorimeter did not deposit enough energy to be confused with direct photons. To aid understanding of the collimator edge effects, a few runs were made in which a special parallelepipedic BGO detector (" $A C$ " counter) was placed in front of the collimator such that $A C$ defined part of the acceptance of the collimator [Fig. 1(c)]. This detector served as an active collimator counter which detected events that should not be accepted.

Behind the collimator was the 4-mm-thick plastic scintillation counter $V$ with PM tubes mounted at opposite ends. This counter detected charged particle background. Since it was also sensitive to backscattered low-energy electrons from the electromagnetic shower developed in the calorimeter, its information was useful in checking the parameters of the shower simulation (see Sec. V).

More details about the photon detector can be found in [13].

\section{Trigger electronics and data acquisition}

The data acquisition was triggered by each of five possible event types: (i) triple coincidence events $A \cdot B \cdot R$ be- 
tween the spectrometer $A$ and $B$ planes and the calorimeter $R$, with the condition that at least one of the 61 counters registered a signal larger than $30 \mathrm{MeV}$; the $A$ threshold was adjusted to eliminate pions, protons, and most of the deuterons from the SPES2 trigger; (ii) "single" events, $(A \cdot B)_{\text {sample }} \cdot \bar{R}$, a sample formed of every 15 th SPES2 event not in coincidence with the calorimeter $\mathrm{R}$; (iii) cosmic rays; (iv) LED events $A \cdot B \cdot R$; (v) electronic generator events which randomly gated the analogue-todigital converters (ADC's) and started the time-to-digital converters (TDC's) in order to provide a measurement of the pileup probability distributions.

During the beam spill, the LED and the electronic generator events were triggered each by one of the beam monitor telescopes, to ensure that they would have the same distribution in time as the $\eta \rightarrow \gamma \gamma$ events. Cosmic rays and uniformly generated LED events were also recorded between beam spills.

The BGO calorimeter counters were connected to ADC's and TDC's. The timing of the TDC starts and ADC gates (190 ns wide) was given by the signal from the SPES2 $A$ plane. The TDC stops required that the calorimeter counter signal be above the $30-\mathrm{MeV}$ threshold, while the ADC's were recorded with a $1-\mathrm{MeV}$ threshold. The complete event information was read from CAMAC registers by a SAR computer [14], using the acquisition program TROLL [15].

\section{EVENT RECONSTRUCTION}

\section{A. Selection of $p d \rightarrow{ }^{3} \mathrm{He} \eta$}

The ${ }^{3} \mathrm{He}$ 's were selected using time-of-flight and pulse height measured with the scintillator planes of SPES2, as discussed in $[9,10]$. The ${ }^{3} \mathrm{He}$ momentum and angle were determined from the SPES2 wire chamber data using a second-order parametrization of trajectories calculated from magnetic field measuremerits.

To select the reaction $p d \rightarrow{ }^{3} \mathrm{He} \eta$, it was convenient to use an expansion of the kinematical variables which is valid in the threshold region [16]. One such variable is:

$$
R_{\mathrm{He}}^{2} \equiv\left(\frac{\delta_{\mathrm{He}}}{1.90}\right)^{2}+\left(\frac{\theta_{x}^{\mathrm{He}}}{17.2}\right)^{2}
$$

where $\theta_{x}^{\text {He }}$ is the ${ }^{3} \mathrm{He}$ horizontal angle, in mrad, while $\delta_{\mathrm{He}} \equiv 100\left(p_{\mathrm{He}}-p_{0}\right) / p_{0}$, and $p_{0}$ is the central value of the ${ }^{3} \mathrm{He}$ momentum $p_{\mathrm{He}}$ expected from kinematics. The cut $R_{\mathrm{He}}^{2}<3.30$ was chosen to accept all the $\eta$ 's. Because the spectrometer resolution of $\theta_{x}^{\mathrm{He}}$ is better than the resolution of the vertical angle $\theta_{y}^{\mathrm{He}}$, it was advantageous to have a separate cut on $\theta_{y}^{\mathrm{He}} ;$ a cut $\left|\theta_{y}^{\mathrm{He}}\right|<50 \mathrm{mrad}$ was used in the selection of $p d \rightarrow{ }^{3} \mathrm{He} \eta$. The precise choice of cuts on the ${ }^{3} \mathrm{He}$ identification, on $R_{\mathrm{He}}^{2}$ and on $\theta_{y}^{\mathrm{He}}$ for selecting $p d \rightarrow{ }^{3} \mathrm{He} \eta$ was unimportant for determining the branching ratio, because the same cuts were used to select $\eta \rightarrow \gamma \gamma$ events (Sec. III B).

A histogram of $R_{\mathrm{He}}^{2}$ with the cut on $\theta_{y}^{\text {He }}$ is shown in Fig. 2. The unshaded histogram represents the single

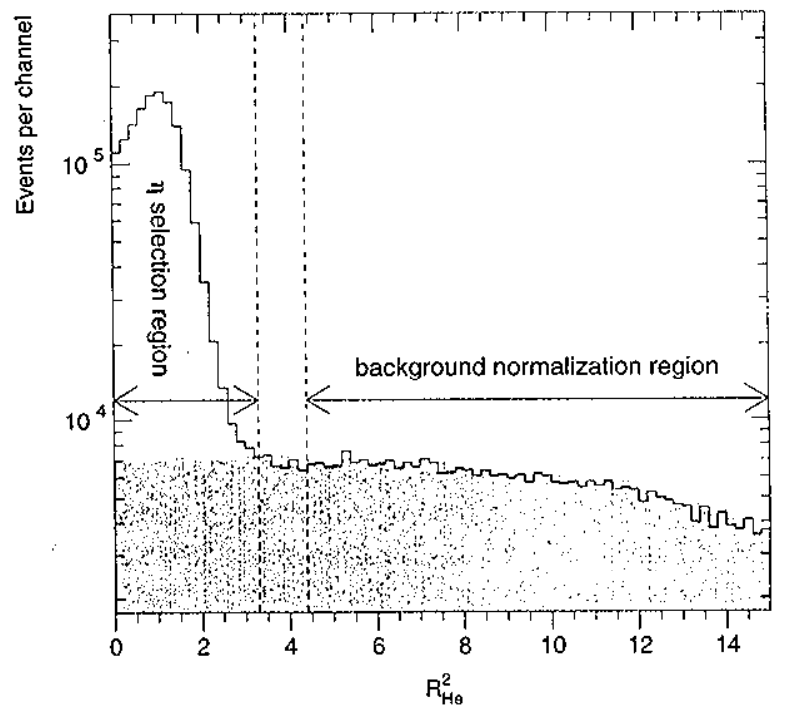

FIG. 2. Histogram of $R_{\mathrm{He}}^{2}$ [Eq. (3)], after applying the ${ }^{3} \mathrm{He}$ identification and $\theta_{y}^{\mathrm{He}}$ cuts (data set 1 ). The peak is due to $p d \rightarrow{ }^{3} \mathrm{He} \eta$ events. The subthreshold data used for the background subtraction (grey shaded area) were normalized in the "background normalization region" of the figure.

events data stream, weighted by a factor 15 to compensate for the sampling of this type of events, augmented by the triple coincidence data stream. This factor 15 was checked within $0.05 \%$ using measured fast scaler information. The number of $p d \rightarrow{ }^{3} \mathrm{He} \eta$ events, $N\left(p d \rightarrow{ }^{3} \mathrm{He} \eta\right)$, was determined from the area of the peak of this histogram after background subtraction. The background under the $\eta$ peak in Fig. 2 was evaluated using two data sets taken at incident beam energies of 2.6 and $4.6 \mathrm{MeV}$ below threshold. The shape of the $R_{\mathrm{He}}^{2}$ distribution was identical for both data sets, as ascertained with a $\chi^{2}$ test which yielded 115 for 150 degrees of freedom. Since, as expected, there was no energy dependence of the background, it was justified to combine both data sets and to use the resulting distribution (grey shaded area in Fig. 2 ) as the best estimate of the background shape for our measurements above threshold.

The systematic uncertainty in the background subtraction was estimated using different normalization regions (see Fig. 2) for the adjustment of the below-threshold sample size to the above-threshold one. This procedure tested in particular for possible local changes in the wire chamber efficiency by selecting physically different areas of the chambers. The total uncertainty in $N\left(p d \rightarrow{ }^{3} \mathrm{He} \eta\right)$ due to the background subtraction; including the statistical uncertainty of the subthreshold measurements, was $0.30 \%$.

\section{B. Selection of $\boldsymbol{\eta} \rightarrow \boldsymbol{\gamma} \boldsymbol{\gamma}$}

The first step in determining the number of $\eta \rightarrow \gamma \gamma$ events, $N(\eta \rightarrow \gamma \gamma)$, was to require that the candidate 
be an $\eta$ produced in the reaction $p d \rightarrow{ }^{3} \mathrm{He} \eta$ as detailed in Sec. III A. Because the same $\eta$ selection cuts were applied to both the $\eta \rightarrow \gamma \gamma$ data stream and the $p d \rightarrow{ }^{3} \mathrm{He} \eta$ data stream, the resulting branching ratio is independent of systematic errors in the beam intensity, the target thickness, and the efficiency of SPES2 for selecting $p d \rightarrow{ }^{3} \mathrm{He} \eta$.

The relative calibration of the pulse heights and timing, and a rough absolute calibration of the pulse heights of all calorimeter counters were achieved using the cosmic ray events, after correcting for the short-term gain variation as monitored by the LED events. The absolute calibration of the pulse heights and timing was done using actual $\eta \rightarrow \gamma \gamma$ events.

To identify a photon in each of the calorimeters, the following algorithm was used. First a search was made for photon candidates, each defined as a calorimeter counter with a larger energy deposit than any of its immediate neighbors. This "central" counter and its six immediate neighbors are referred to as a "cluster" for the following discussion. The typical probability of observing zero, one, two, or three photon candidates was $71 \%, 24 \%, 4 \%$, and $0.5 \%$, respectively, in the calorimeter $L$, and $0,98 \%$, $2 \%$, and $0.05 \%$, respectively, in the calorimeter $R$. The multiple identification may be due to the presence of an additional photon or other particle hitting the detector, or it may be due to a false identification. In case multiple photon candidates were identified, each left-right combination was considered for the $\eta \rightarrow \gamma \gamma$ selection criteria discussed below. Whenever at least one combination passed all the $\eta \rightarrow \gamma \gamma$ selection criteria, $N(\eta \rightarrow \gamma \gamma)$ was incremented by one. By analyzing all left-right combinations rather than just one, $0.6 \%$ more $\eta \rightarrow \gamma \gamma$ events were recovered.

The photon timing was determined from the timing of the central counter, with a resolution $\sigma_{t}=0.4 \mathrm{~ns}$. Prompt triple coincidences were selected by requiring $\left|\Delta t_{L-\mathrm{He}}\right|<2$ ns and $\left|\Delta t_{R-\mathrm{He}}\right|<2$ ns, where $\Delta t_{L-\mathrm{He}}$ and $\Delta t_{R-H e}$ are the left and right calorimeter times with respect to the SPES2 $A$ plane, centered at zero for $\eta \rightarrow \gamma \gamma$ events. The photon horizontal and vertical angles were determined using an algorithm which made use of the sharing of the electromagnetic shower energy among the counters in the cluster. The resolution of the vertical and horizontal angles was $\sigma_{\theta_{X}} \sim \sigma_{\theta_{Y}} \sim 0.7^{\circ}$ for each photon. The photon energy was determined by summing all the pulse heights for all calorimeter counters which had timing either within a $6 \mathrm{~ns}$ window of the central counter or earlier or had no timing information at all.

With this method the photon energy was not underestimated. However, in events with pileup, the photon energy may have been overestimated. Since no upper window cut was applied to the photon energies, overestimating the photon energy did not lead to the rejection of valid $\eta \rightarrow \gamma \gamma$ events. The energy resolution, $\sigma_{E} / E \sim 4 \%$, sufficed for this experiment but was not optimal for this type of calorimeter because of the procedure used in the energy reconstruction. Further details on determining the photon timing, angle, and energy are given in Ref. [13].

Energy conservation was tested by requiring

$$
\Delta E \equiv E_{p}+M_{d} c^{2}-\left(E_{\mathrm{He}}+E_{L}+E_{R}\right)<100 \mathrm{MeV},
$$

where $E_{p}$ and $E_{\mathrm{He}}$ are the energies of the proton and ${ }^{3} \mathrm{He}$, $M_{d}$ is the deuteron mass and $E_{L}$ and $E_{R}$ are the left and right photon energies. $\Delta E$ is zero for photons from $\eta \rightarrow$ $\gamma \gamma$. Figure 3 shows a histogram of $\Delta E$. Because of the way in which $E_{L}$ and $E_{R}$ were calculated, random pileup added high-energy tails to $E_{L}$ and $E_{R}$ which shows up as a tail at the low end of the $\Delta E$ histogram. It was desirable for the cut on $\Delta E$ not to reject events which were pushed into this tail by random pileup. Therefore no lower cutoff was applied to $\Delta E$.

In addition to cutting background, the cut on $\Delta E$ also discriminated against almost all the $\eta \rightarrow \gamma \gamma$ events in which one photon hit the collimator (see Fig. 3). The remaining events of this type were removed by the subsequent constraints $E_{R}>200 \mathrm{MeV}$ and $E_{L}>200 \mathrm{MeV}$.

Another test made use of the expected angular correlation between the two photons:

$$
\begin{array}{r}
\left|\Delta \theta_{L R}\right| \equiv\left|\theta_{L R}^{\text {calc }}-\theta_{L R}^{\text {meas }}\right|<5^{\circ}, \\
\text { with } \cos \left(\frac{1}{2} \theta_{L R}^{\text {calc }}\right)=\beta_{\eta}\left[1-\frac{1}{8}\left(\theta_{L}-\theta_{R}\right)^{2}\right],
\end{array}
$$

where $\theta_{L R}^{\text {meas }}$ is the two-photon opening angle as measured by the calorimeters and $\theta_{L R}^{\text {calc }}$ is the expected $\eta \rightarrow \gamma \gamma$ opening angle calculated from the $\eta$ velocity $\left(\beta_{\eta}\right)$, as deduced from the measured ${ }^{3} \mathrm{He}$ vector momentum. The quantities $\theta_{L}$ and $\theta_{R}$ are the left and right photon angles with respect to the $\eta$ direction, expressed in radians.

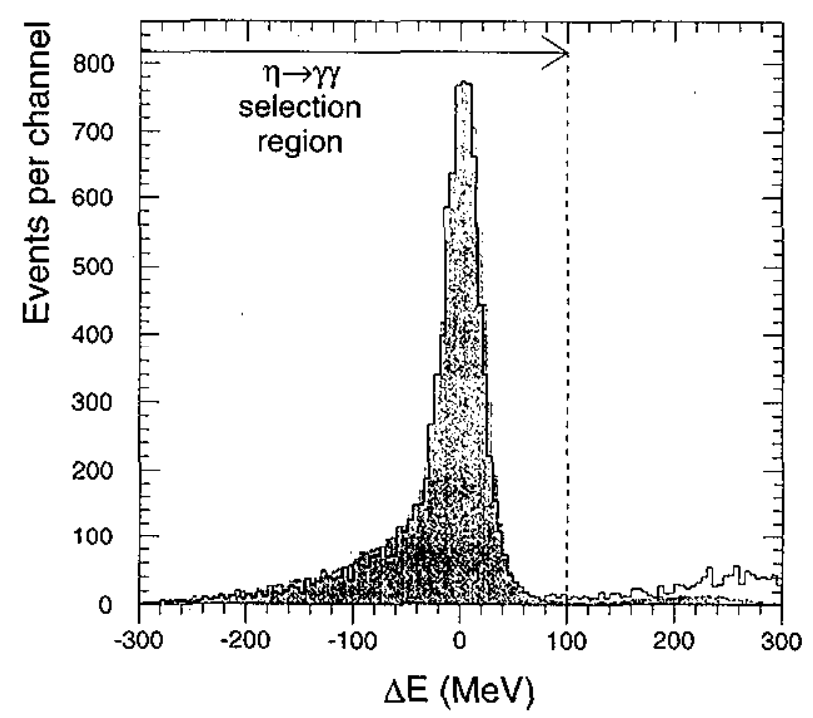

FIG. 3. Histogram of the energy correlation $\Delta E$ [Eq. (4)] after applying all cuts of Table I except the energy cuts. The solid line is for data set 1 . The grey shaded histogram is for simulated $\eta \rightarrow \gamma \gamma$ events. The cut $\Delta E<100 \mathrm{MeV}$ is illustrated with a dashed line. The background appearing above the cut is composed of $\eta \rightarrow \gamma \gamma$ events with a photon hitting the collimator and of other $\eta$ decay channels which were not taken into account in the simulation. 
The term proportional to $\left(\theta_{L}-\theta_{R}\right)^{2}$ is a correction which applies to the slightly asymmetric decays measured in this experiment. For $\eta \rightarrow \gamma \gamma$ decays, the distribution of $\Delta \theta_{L R}$ was centered at zero and had a Gaussian shape with $\sigma \simeq 1.1^{\circ}$ [Fig. 4(c)]. This distribution identified the $\eta \rightarrow \gamma \gamma$ decays. Background from $p d \rightarrow{ }^{3} \mathrm{He} \pi^{+} \pi^{-}$ and from two-body $\eta$ decays in which the decay products are massive would peak at $\Delta \theta_{L R}>0$ because of the smaller opening angles, and other background would be a continuum.

Table I summarizes the cuts used to select $\eta \rightarrow \gamma \gamma$ events. Figure 4 shows the spectrum of $\Delta \theta_{L R}$ at various stages of the analysis. The final spectrum of $\Delta \theta_{L R}$ after all cuts contains only a small background, of the order of $0.3 \%$. The continuum background is due to other $\eta$ decay modes and $p d \rightarrow{ }^{3} \mathrm{He} \pi^{+} \pi^{-}$events in combination with random coincidences. The background subtraction was performed in three stages. First random coincidences were determined from events which failed the $\eta \rightarrow \gamma \gamma$

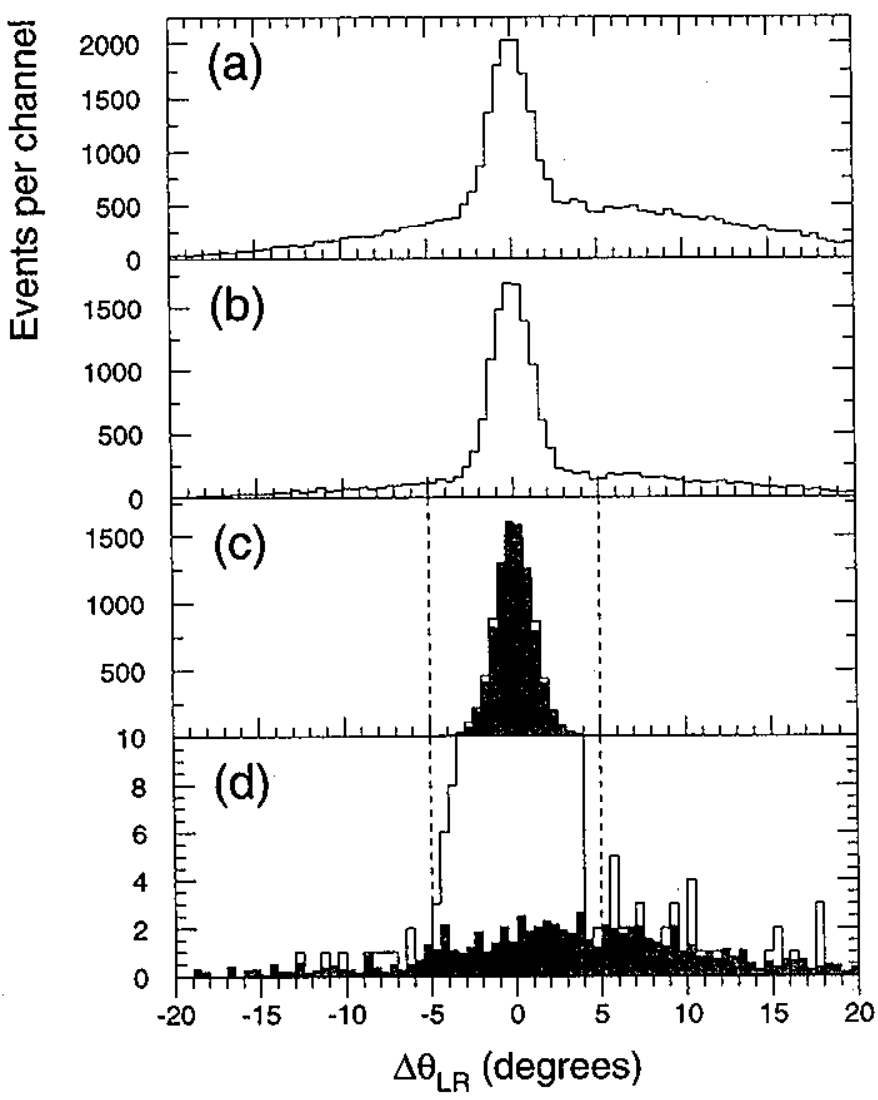

FIG. 4. Histogram of the angular correlation $\Delta \theta_{L R}$ [Eq. (5)] at various stages of the analysis: (a) only the cuts used to select $p d \rightarrow{ }^{3} \mathrm{He} \eta$ applied; (b) same, plus calorimeter timing cuts applied; (c) all standard cuts (listed in Table I) applied except the cut on $\Delta \theta_{L R}$ (the dashed vertical lines illustrate the cut $\left|\Delta \theta_{L R}\right|<5^{\circ}$ and the grey shaded area corresponds to simulated data); (d) enlarged version of (c) showing the minimal background outside the $\eta \rightarrow \gamma \gamma$ peak; the grey shaded histogram is the estimated background.
TABLE I. Summary of the $\eta \rightarrow \gamma \gamma$ selection cuts used in the analysis.

\begin{tabular}{c}
\hline Cuts to select $\eta \rightarrow \gamma \gamma$ \\
${ }^{3}$ He identification \\
$R_{\mathrm{He}}^{2}<3.30$ \\
$\left|\theta_{y}^{\mathrm{He}}\right|<50 \mathrm{mrad}$ \\
$\left|\Delta t_{L-\mathrm{He}}\right|<2 \mathrm{~ns}, \quad\left|\Delta t_{R-\mathrm{He}}\right|<2 \mathrm{~ns}$ \\
$\Delta E<100 \mathrm{MeV}$ \\
$E_{L}>200 \mathrm{MeV}, \quad E_{R}>200 \mathrm{MeV}$ \\
$\left|\Delta \theta_{L R}\right|<5^{\circ}$ \\
\hline
\end{tabular}

timing selection test. This accounted for about $2 / 5$ of the background. Then events which failed the identification of the production reaction were normalized by the ratio of acceptances of $p d \rightarrow{ }^{3} \mathrm{He} X$ within and outside the $\eta$ selection region, as determined by the subthreshold data. These events were of the type $p d \rightarrow{ }^{3} \mathrm{He} X$, where $X$ may be any particle or combination of particles other than the $\eta$, most probably 2 or 3 pions, but also possibly 2 photons (see Sec. IIIC). They accounted for another $1 / 5$ of the background. The shape of the remaining background was assumed to be the same as events with $100 \mathrm{MeV}<\Delta E<200 \mathrm{MeV}$, coming predominantly from $\eta \rightarrow 3 \pi$ decays. The final result was, however, not sensitive to the exact shape assumed for this final background subtraction. The systematic uncertainty on $N(\eta \rightarrow \gamma \gamma)$ associated with the total background subtraction was estimated to be $0.12 \%$.

\section{Search for correlated background from $p d \rightarrow{ }^{3} \mathrm{He} \gamma \gamma$}

As just mentioned, data outside of the allowed kinematics region of $p d \rightarrow{ }^{3} \mathrm{He} \eta$ were selected to search for $p d \rightarrow{ }^{3} \mathrm{He} \gamma \gamma$ or $p d \rightarrow{ }^{3} \mathrm{He} X$ followed by $X \rightarrow \gamma \gamma$, where $X$ is any particle other than the $\eta$. The search was sensitive to $520 \mathrm{MeV}<M_{X}<546 \mathrm{MeV}$. Because it is a second-order electromagnetic interaction, direct two-photon production by $p d \rightarrow{ }^{3} \mathrm{He} \gamma \gamma$ is expected to be suppressed by a factor $\frac{1}{\alpha^{2}}$ compared to $p d \rightarrow{ }^{3} \mathrm{He} \eta$ and is not expected to be seen here.

For this search, $\Delta \theta_{L_{R}}$ was calculated using the missing mass $M_{X}$ which was calculated from the ${ }^{3} \mathrm{He}$ measured momentum rather than assuming the $\eta$ mass; therefore such background events would have $\Delta \theta_{L R}=0$. There was no evidence of a two-photon peak at $\Delta \theta_{L R}=0$. If such background existed, it would have been incorporated into the second background subtraction discussed above. No further adjustment was made to the number of $\eta \rightarrow \gamma \gamma$ events.

\section{SIMULATION OF THE EXPERIMENT}

A detailed simulation of the experiment was needed to determine the acceptance and the reconstruction efficiency for the $\eta \rightarrow \gamma \gamma$ events. The reaction $p d \rightarrow{ }^{3} \mathrm{He} \eta$ 
followed by $\eta \rightarrow \gamma \gamma$ was simulated using GEANT [17]. The simulation included the phase-space distribution of the incident proton beam. The $\eta$ mesons (and the ${ }^{3} \mathrm{He}$ ) were generated isotropically in the center-of-mass frame. This isotropic distribution has been verified with real $p d \rightarrow{ }^{3} \mathrm{He} \eta$ events $[10,16]$ (an anisotropy would affect the $\eta \rightarrow \gamma \gamma$ acceptance). The ${ }^{3} \mathrm{He}$ momentum and angle resolutions resulting from multiple scattering and energy loss in the target and in other materials, as well as from the spectrometer intrinsic resolution, were approximated by Gaussian distributions. The photons from $\eta \rightarrow \gamma \gamma$ and the subsequent electromagnetic showers were tracked through the collimator and the calorimeters. All materials in the paths between the target and the calorimeters were considered. Photon conversion before the calorimeters occurred mostly in the $V$ counter, with a probability of about $1 \%$. The intrinsic resolution of each BGO counter was approximated with a Gaussian distribution.

The $\eta \rightarrow \gamma \gamma$ detector acceptance $\mathcal{A}_{\eta \rightarrow \gamma \gamma}$ was determined using the simulated $\eta \rightarrow \gamma \gamma$ events which were accepted by the calorimeters with the constraints $E_{L}>$ $200 \mathrm{MeV}$ and $E_{R}>200 \mathrm{MeV}$. For the nominal run conditions (data set 1), $\mathcal{A}_{\eta \rightarrow \gamma \gamma}=0.01516 \pm 0.00007$ (stat).

An accurate account of the effect of random pileup on the analysis efficiency was achieved by directly incorporating into the ADC and TDC values of the simulated data the background observed in the electronic generator events. The $\eta \rightarrow \gamma \gamma$ selection efficiency $\epsilon_{\eta \rightarrow \gamma \gamma}^{\text {analysis }}$ is given by the fraction of simulated, accepted $\eta \rightarrow \gamma \gamma$ events which pass the $\eta \rightarrow \gamma \gamma$ selection criteria (Table I).

For most of the data sets, $\epsilon_{\eta \rightarrow \gamma \gamma}^{\text {analysis }} \sim 0.996$. The uncertainty of $\mathcal{A}_{\eta \rightarrow \gamma \gamma}$ and $\epsilon_{\eta \rightarrow \gamma \gamma}^{\text {analysis }}$ will be discussed in Sec. V. More details of the simulation can be found in Ref. [13].

\section{DETERMINATION OF THE $\eta \rightarrow \gamma \gamma$ BRANCHING RATIO}

The $\eta \rightarrow \gamma \gamma$ branching ratio was obtained using the expression

$$
\begin{aligned}
B(\eta \rightarrow \gamma \gamma)= & \frac{N(\eta \rightarrow \gamma \gamma)}{N\left(p d \rightarrow{ }^{3} \mathrm{He} \eta\right)} \\
& \times\left(\mathcal{A}_{\eta \rightarrow \gamma \gamma} \epsilon_{\eta \rightarrow \gamma \gamma}^{\text {analysis }} \epsilon_{\eta \rightarrow \gamma \gamma}^{\text {electronics }}\right)^{-1},
\end{aligned}
$$

where $N(\eta \rightarrow \gamma \gamma)$ is the number of $\eta \rightarrow \gamma \gamma$ events (Sec. III B), $N\left(p d \rightarrow{ }^{3} \mathrm{He} \eta\right)$ is the number of tagged $\eta$ (Sec. III A), $\mathcal{A}_{\eta \rightarrow \gamma \gamma}$ is the detector acceptance for $\eta \rightarrow \gamma \gamma$ (Sec. IV), $\epsilon_{\eta \rightarrow \gamma \gamma}^{\text {analysis }}$ is the efficiency of the $\eta \rightarrow \gamma \gamma$ event selection (Sec. IV), and $\epsilon_{\eta \rightarrow \gamma \gamma}^{\text {electronics }}$ is discussed below. Table II lists these quantities for the eight data sets.

The fractional loss of triple coincidence events due to the calorimeter electronics $\left(1-\epsilon_{\eta \rightarrow \gamma \gamma}^{\text {electronics }}\right)$ was dominated by an occasional failure of the ADC readout system, which was detectable. All electronic components and modules were tested by the LED events. For the nominal run conditions (dat'a set 1 ), $\epsilon_{\eta \rightarrow \gamma \gamma}^{\text {electronics }}=0.998 \pm 0.002$.

The branching ratio was averaged over the eight data sets. The result from each data set $i$ was weighted by $1 / \sigma_{i}^{2}$, where $\sigma_{i}$ is the statistical uncertainty associated with data set $i$, including the statistical error in the simulated acceptance. The branching ratio averaged over the eight data sets is $B(\eta \rightarrow \gamma \gamma)=0.3949 \pm 0.0017$ (stat). The agreement of the 108 data taking runs comprising these eight different experimental conditions provided further evidence of the good internal consistency of the data [13].

The remainder of this section contains a discussion of systematic errors common to all the data sets.

The uncertainties on $N\left(p d \rightarrow{ }^{3} \mathrm{He} \eta\right)$ and $N(\eta \rightarrow \gamma \gamma)$ have been discussed in Secs. III A and III B.

The uncertainty in the beam position was estimated by examining the recoil ${ }^{3} \mathrm{He}$. A shift in the beam position or angle results in a shift in the ${ }^{3} \mathrm{He}$ angle spectrum at the SPES2 focal plane. The average beam position is known to better than $0.75 \mathrm{~mm}$, which is dominated by the uncertainties in the positioning of the wire chambers used to align the beam between runs. While drifts of $\pm 1.5 \mathrm{~mm}$ in the beam horizontal position did occur, such drifts were unimportant because the acceptance depended linearly on the beam horizontal position to first

TABLE II. Analysis results of the 8 data sets. See Sec. V for an explanation of the analysis quantities. The collimator aperture angle $\alpha$ [Fig. 1(c)] and the distances $d_{L}$ and $d_{R}$ from the target to the calorimeters are also given. The uncertainties

\begin{tabular}{|c|c|c|c|c|c|c|c|c|}
\hline Data set & 1 & 2 & 3 & 4 & 5 & 6 & 7 & 8 \\
\hline$d_{L}(\mathrm{~mm})$ & 653 & 728 & 547 & 653 & 728 & 728 & 653 & 728 \\
\hline$d_{R}(\mathrm{~mm})$ & 883 & 883 & 883 & 776 & 979 & 979 & 979 & 979 \\
\hline$\alpha$ & $18^{\circ}$ & $18^{\circ}$ & $18^{\circ}$ & $18^{\circ}$ & $15^{\circ}$ & $18^{\circ}$ & $18^{\circ}$ & $12^{\circ}$ \\
\hline \multirow[t]{2}{*}{$N(\eta \rightarrow \gamma \gamma)$} & 8787 & 4391 & 8885 & 9058 & 7216 & 9081 & 8616 & 8712 \\
\hline & \pm 94 & \pm 66 & \pm 94 & \pm 95 & \pm 85 & \pm 96 & \pm 93 & \pm 94 \\
\hline \multirow{2}{*}{$\begin{array}{l}N\left(p d \rightarrow{ }^{3} \mathrm{He} \eta\right) \\
\left(\times 10^{6}\right)\end{array}$} & 1.472 & 0.741 & 1.502 & 1.519 & 1.758 & 1.544 & 1.459 & $3 . \overline{357}$ \\
\hline & \pm 0.005 & \pm 0.003 & \pm 0.005 & \pm 0.005 & \pm 0.005 & \pm 0.005 & \pm 0.005 & \pm 0.007 \\
\hline $\mathcal{A}_{\eta \rightarrow \gamma \gamma}$ & 0.0152 & 0.0149 & 0.0152 & 0.0151 & 0.0104 & 0.0151 & 0.0150 & 0.0067 \\
\hline$\epsilon_{\eta \rightarrow \gamma \gamma}^{\text {analysis }}$ & 0.997 & 0.996 & 0.995 & 0.996 & 0.996 & 0.995 & 0.996 & 0.996 \\
\hline $\begin{array}{l}\text { electronics } \\
\epsilon_{\eta \rightarrow \gamma \gamma}^{\text {ele }}\end{array}$ & 0.998 & 0.998 & 0.995 & 0.995 & 0.996 & 0.996 & 0.997 & 0.996 \\
\hline \multirow[t]{2}{*}{$\overline{B(\eta \rightarrow \gamma \gamma)}$} & 0.396 & 0.399 & 0.392 & 0.397 & 0.396 & 0.392 & 0.395 & 0.393 \\
\hline & \pm 0.005 & \pm 0.007 & \pm 0.005 & \pm 0.005 & \pm 0.005 & \pm 0.005 & \pm 0.005 & \pm 0.005 \\
\hline
\end{tabular}
given for $N(\eta \rightarrow \gamma \gamma), N\left(p d \rightarrow{ }^{3} \mathrm{He} \eta\right)$, and $B(\eta \rightarrow \gamma \gamma)$ are statistical only. 
order. The uncertainty in the beam direction does not affect significantly the uncertainty in the acceptance.

The uncertainty in the beam energy includes a $0.1 \mathrm{MeV}$ uncertainty in determining the absolute beam energy and a $0.2 \mathrm{MeV}$ allowance for small drifts.

The $0.36 \%$ uncertainty in the detector acceptance due to the collimator placement and aperture size is broken down as follows: $0.2 \%$ due to the $0.1 \%$ uncertainty in the collimator aperture size, $0.25 \%$ due to the $0.8 \mathrm{~mm}$ uncertainty in the distance from the target to the collimator, and $0.17 \%$ due to the $0.1^{\circ}$ uncertainty in the detector axis direction.

The uncertainty in $\epsilon_{\eta \rightarrow \gamma \gamma}^{\text {analysis }}$ due to uncertainties in the simulation and the detector calibration was estimated using several variations on the analysis:

(1) A signal in the $V$ counter in time with the $R$ calorimeter could come from charged particle background (e.g., $p d \rightarrow{ }^{3} \mathrm{He} \pi^{+} \pi^{-}$), from photon conversion, or from backward leakage from the calorimeter shower. When the $V$ counter was used as an anticoincidence, the observed rejection of $3.4 \%$ of the $\eta \rightarrow \gamma \gamma$ events (in data set 1) was, however, due in part to accidental pileup in this counter. This figure was anticipated by the simulation, so that the branching ratio from this analysis agreed with the standard analysis within $0.16 \%$.

(2) The $A C$ counter was placed as in Fig. 1(c) for some special runs and used in anticoincidence in the corresponding analysis, to test our understanding of the collimator edge effects. The observed rejection of $0.7 \%$ of the $\eta \rightarrow \gamma \gamma$ events was anticipated by the simulation, but again dominated by pileup in the counter $A C$. The branching ratio from this analysis agreed with the standard analysis within $0.18 \%$.

(3) An alternate pileup measurement was performed: pileup was incorporated into the simulation using the LED events instead of the electronic generator events. The amplitude and timing contributions expected from the LED's were subtracted out. This analysis tested the method of incorporating the pileup into the simulation. The branching ratio from this analysis agreed with the standard analysis within $0.18 \%$.

(4) The simulated detector response was changed to allow for possible discrepancies between the simulated and real detector responses. Photon energies were scaled by $3 \%$, timing distributions were widened by $15 \%$, and the distribution of $\Delta \theta_{L R}$ was widened by $10 \%$. The branching ratio from this analysis agreed with the standard analysis within $0.17 \%$.

(5) A second analysis approach was applied to data sets 4 and 8 . This analysis used independent methods of selecting the ${ }^{3} \mathrm{He}$, reconstructing the ${ }^{3} \mathrm{He}$ trajectories, and selecting $p d \rightarrow{ }^{3} \mathrm{He} \eta$ events. The photon energies, timing, and angles were reconstructed from the local cluster of seven BGO elements only (a central element and its six nearest neighbors). The algorithm used for angle reconstruction was based on a neural network approach [18]. The selection of $\eta \rightarrow \gamma \gamma$ events was based on a kinematic fit to all free variables and a $10 \sigma$ cut on the resulting $\chi^{2}$ distribution. The background was estimated from the shape of the $\chi^{2}$ distribution at large $\chi^{2}$ extrapolated to the signal region. This analysis was more efficient than the standard analysis at rejecting background events and at selecting $p d \rightarrow{ }^{3} \mathrm{He} \eta$. However, due to the tight $\eta \rightarrow \gamma \gamma$ selection criteria, it was more sensitive to the effects of pileup and to the simulation parameters. Because this analysis was sensitive to systematic effects differently than the standard one, a comparison of the two analyses verified the understanding of the most important aspects of the experiment: the simulation of collimator edge effects and electromagnetic showering in the detectors, the calibration of the experiment and the effects of random pileup. Although some factors in Eq. (6) differed in the two analyses by as much as $3 \%$, the resulting branching ratios agreed within $0.17 \%$.

All the variations in the above analyses are not independent. For example, analyses (1), (2), and (5) all

TABLE III. Systematic uncertainties on $B(\eta \rightarrow \gamma \gamma)$.

\begin{tabular}{lcc}
\hline \hline Source of the systematic uncertainty & $\begin{array}{c}\text { Quantity } \\
\text { affected }\end{array}$ & $\begin{array}{c}\text { Relative } \\
\text { uncer- } \\
\text { tainty }\end{array}$ \\
\hline $\begin{array}{l}\eta \gamma \gamma \text { background subtraction } \\
\text { uncertainty }\end{array}$ & $N(\eta \rightarrow \gamma \gamma)$ & $0.12 \%$ \\
\hline $\begin{array}{l}\eta \text { background subtraction uncertainty } \\
\text { Uncertainty on sampling factor }\end{array}$ & $N\left(p d \rightarrow{ }^{3} \mathrm{He} \eta\right)$ & $0.30 \%$ \\
\hline 0.75 mm uncertainty in beam position & $\mathcal{A}_{\eta \rightarrow \gamma \gamma}$ & $0.05 \%$ \\
$\begin{array}{l}\text { Beam energy fluctuations } \\
\text { Uncertainty in the collimator }\end{array}$ & $0.27 \%$ \\
$\quad$ aperture size and position & & $0.26 \%$ \\
$\quad$ mm uncertainty in target position & & $0.36 \%$ \\
Possible anisotropy in $\eta$ production & & $0.18 \%$ \\
\hline Accuracy of the simulation & $\epsilon_{\eta \rightarrow \gamma \gamma}^{\text {analysis }}$ & $0.17 \%$ \\
\hline The electronics & $\epsilon_{\eta \rightarrow \gamma \gamma}^{\text {electronics }}$ & $0.28 \%$ \\
\hline Total (in quadrature) & & $0.22 \%$ \\
\hline \hline
\end{tabular}


tested in part the validity of the treatment of pileup effects, especially addressed in analysis (3); analyses (4) and (5) both tested the simulation of the electromagnetic showers; finally, analyses (2) and (5) both investigated the collimator edge effects. The resulting estimate of the systematic uncertainty in $\epsilon_{\eta \rightarrow \gamma \gamma}^{\text {analysis }}$ is $0.28 \%$.

There was an unexplained phenomenon in the electronics in which a small number of events $(0.4 \%$ relative to the sum of single and triple coincidence events) were labeled as both triple coincidence events and single events. For the analysis, it was unknown whether to treat these events as single events which should be weighted to compensate for the sampling or as triple coincidence events which should not be weighted. A relative uncertainty of $0.22 \%$ resulted from this problem.

A total systematic uncertainty of $0.75 \%$ was obtained as the quadratic sum of all uncertainties discussed above, and listed in Table III.

\section{RESULTS}

Based on $(6.47 \pm 0.03) \times 10^{4}$ clean $\eta \rightarrow \gamma \gamma$ events, the first direct, precision measurement of the branching ratio for $\eta \rightarrow \gamma \gamma$ resulted in

$$
B(\eta \rightarrow \gamma \gamma)=0.3949 \pm 0.0017(\text { stat }) \pm 0.0030(\text { syst })
$$

This result is consistent with the branching ratio obtained by indirect measurements [6] and is free from the systematic uncertainties associated with these previous measurements.

The present result, when used in conjunction with other experiments, allows to set an upper limit on all neutral $\eta$ decay modes, $X_{0}$, not observed so far. By definition,

$$
\begin{aligned}
B\left(X_{0}\right)= & B(\eta \rightarrow \text { neutrals })-B(\eta \rightarrow \gamma \gamma) \\
& \times\left[1+\frac{\Gamma\left(\eta \rightarrow 3 \pi^{0}\right)}{\Gamma(\eta \rightarrow \gamma \gamma)}+\frac{\Gamma\left(\eta \rightarrow \pi^{0} \gamma \gamma\right)}{\Gamma(\eta \rightarrow \gamma \gamma)}\right]
\end{aligned}
$$

All quantities on the right side of Eq. (8) are experimentally measured (Refs. $[7,8]$ and this work). Substituting the experimental results, one finds $B\left(X_{0}\right)=$ $-0.015 \pm 0.011$ for this positive definite quantity. Using the most conservative method suggested in Ref. [6] (p. 1280 , option 3 ), the following upper limit is deduced, at $90 \%$ confidence level:

$$
B\left(X_{0}\right)<2.8 \%
$$

This implies an upper limit of $2.8 \%$ for the branching ratios of the $P$-and $C P$-violating decay $\eta \rightarrow \pi^{0} \pi^{0}$, of the $C$-violating decay $\eta \rightarrow \pi^{0} \pi^{0} \gamma$, and of other exotic decay modes into neutrals.

\section{ACKNOWLEDGMENTS}

The authors wish to thank the SATURNE staff for the high quality beam and the technical support received during the course of the experiment. They would like to thank P. Chesny, G. Guillier, and J.M. Gheller for building and handling the cryogenic target, J.P. Mouly and A. Zaccharian for technical help, R. Kessler and M. Rouger for their assistance in the experimental design, and J. Prokop and A. Tong for their participation in the data taking and the calibration, respectively. Finally, the authors wish to express gratitude to the PaulScherrer-Institut for the generous loan of its quality BGO calorimeters and support electronics, and to U. Moser for the technical information concerning these calorimeters. This research was supported in part by the Commissariat à l'Energie Atomique, the U.S. Department of Energy, the U.S. National Science Foundation, and the North Atlantic Treaty Organization.
[1] S. Adler, Phys. Rev. 177, 2426 (1969); J. Bell and R. Jackiw, Nuovo Cimento A 60, 47 (1969).

[2] F. Gilman and R. Kaufman, Phys. Rev. D 36, 2761 (1987).

[3] G. Shore and G. Veneziano, Nucl. Phys. B381, 3 (1992).

[4] G. Kozlov (private communication).

[5] N. Roe, in Ref. [6], p. 1451.

[6] Particle Data Group, L. Montanet et al., Phys. Rev. D 50, 1173 (1994).

[7] M. Basile et al., Nuovo Cimento A 3, 796 (1971).

[8] D. Alde et al., Sov. J. Nucl. Phys. 40, 918 (1984).

[9] R. Abegg et al., Phys. Rev. D 50, 92 (1994).

[10] B. Mayer et al., Report No. CEA/DAPNIA/SPhN 95-4, 1995, submitted to Phys. Rev. C.

[11] G. Czapek et al., Phys. Rev. Lett. 70, 17 (1993); and C. Hug, Ph.D. thesis, Universität Bern, 1989.

[12] G. Gervino et al., Nucl. Instrum. Methods A309, 497
(1991).

[13] D. White, Ph.D. thesis, University of California, Los Angeles, 1994; and UCLA. Internal Report ETA-45, 1995 (unpublished).

[14] B. Bricaud, J. Faivre, and J. Pain, IEEE Trans. Nucl. Sci. NS-26, 4641 (1979).

[15] L. Roussier and L. Farvacque, Report No. LNS SSG 83.19 /INEL 142, 1983 (unpublished).

[16] R. Kessler, Ph.D. thesis, University of California, Los Angeles, 1992.

[17] CERN Program Library Long Writeup W5013, version 3.16, 1993.

[18] P. Fuchs, in Proceedings of the Conference on Computing in High Energy Physics, San Francisco, 1994, edited by S.C. Loken [Report No. LBL-35822, p. 504 (unpublished)]. 